\title{
Cavity-mediated coupling of mechanical oscillators limited by quantum back-action
}

\author{
Nicolas Spethmann ${ }^{1,2 \star}$, Jonathan Kohler ${ }^{1}$, Sydney Schreppler ${ }^{1}$, Lukas Buchmann ${ }^{1}$ \\ and Dan M. Stamper-Kurn ${ }^{1,3}$
}

\begin{abstract}
A complex quantum system can be constructed by coupling simple elements. For example, trapped-ion ${ }^{1,2}$ or superconducting $^{3}$ quantum bits may be coupled by Coulomb interactions, mediated by the exchange of virtual photons. Alternatively, quantum objects can be made to emit and exchange real photons, providing either unidirectional coupling in cascaded geometries $^{4-6}$, or bidirectional coupling that is particularly strong when both objects are placed within a common electromagnetic resonator ${ }^{7}$. However, in such an open system, the capacity of a coupling channel to convey quantum information or generate entanglement may be compromised by photon $\operatorname{loss}^{8}$. Here, we realize phase-coherent interactions between two addressable, spatially separated, near-groundstate mechanical oscillators within a driven optical cavity. We observe the quantum back-action noise imparted by the optical coupling resulting in correlated mechanical fluctuations of the two oscillators. Our results illustrate challenges and opportunities of coupling quantum objects with light for applications of quantum cavity optomechanics ${ }^{8-14}$.
\end{abstract}

Cavity optomechanical systems comprised of a single mechanical oscillator interacting with a single electromagnetic cavity mode ${ }^{15}$ serve useful quantum-mechanical functions, such as generating squeezed light ${ }^{16-18}$, detecting forces with quantum-limited sensitivity ${ }^{19}$ or through back-action-evading measurement ${ }^{20}$, and both entangling and amplifying mechanical and optical modes ${ }^{21}$. Systems containing several mechanical elements offer additional capabilities. In the quantum regime, these systems may enable two-mode back-action-evading measurements ${ }^{9}$, creation of nonclassical states ${ }^{10}$, fundamental tests of quantum mechanics ${ }^{8,11,12}$, correlations at the quantum level with applications in highsensitivity measurements ${ }^{13}$, and quantum information science ${ }^{14}$. Realizing these proposed functions requires multiple-element cavity optomechanical systems in which quantum-mechanical optical force fluctuations dominate over thermal and technical ones.

An important new feature in these multi-mechanical systems is photon-mediated forces between mechanical elements. Consider a driven cavity containing two mechanical elements with linear optomechanical coupling (Fig. 1a). Each element experiences radiation pressure proportional to the number of intracavity photons. The displacement of one element changes the cavity resonance frequency, causing a change in the intracavity photon number, and thereby modifying the force on the second element. In this manner, an effective optical spring is established between the mechanical elements (Fig. 1b). A quantized picture clarifies the role of cavity photons as the bidirectional force-mediating particle for this interaction: pump light is Stokes scattered off one element, generating a cavity photon that is absorbed through anti-Stokes scattering by the second element, and vice versa ${ }^{7}$ (Fig. 1c). This cavity-mediated force has been shown to cause hybridization ${ }^{22-24}$ and synchronization ${ }^{25}$ of, and incoherent transfer of energy between ${ }^{24}$, mechanical modes. Cavity-mediated longrange interactions are also exhibited by the mechanical instability of an atomic gas within a transversely pumped optical cavity ${ }^{26,27}$.

However, in such an optically driven system, the cavity photons not only mediate the two-way interaction, but also act as information carriers: The light emitted from the cavity permits measurements on the mechanical elements inside the cavity, leading to measurement back-action noise and limiting the capacity of cavity-mediated interactions to transfer quantum states between the coupled elements ${ }^{8}$. Thermal and technical noise prevented the observation of such incoherent back-action in previous optomechanical experiments. In this work, we focus on light-mediated interactions between two objects placed within a common high-finesse optical resonator, a geometry that promises some immunity against the deleterious effects of the loss of the interaction mediating photons. Light-mediated interactions have also been realized in cascaded geometries ${ }^{4,5,28}$, which provide only unidirectional communication. Back-action effects in such systems, inevitably imposed by photon loss, may be circumvented by postselection-that is, examining only experimental realizations that are heralded by a photodetection event ${ }^{6}$. In contrast, our work specifically aims to identify this incoherent cavity-mediated backaction force, and hence examines the complete and unconditioned data record.

Here, we study a cavity optomechanical system containing two non-degenerate, spatially separated and well-isolated mechanical oscillators, each comprised of a trapped ultracold gas of ${ }^{87} \mathrm{Rb}$ atoms. Applying cavity pump light that couples the mechanical elements, we observe light-induced forces through the redistribution of optomechanical cooperativity and, thereby, of radiation-pressure quantum noise between the different modes of motion. In addition, we perform time-resolved measurements of the mechanical states of the two oscillators, with sensitivity near the standard quantum limit, to reveal the phase-coherent, oscillatory exchange of energy between the elements, and also the build-up of incoherent back-action noise that accompanies the cavity-mediated coupling.

Following previous work $^{29}$, we trap around 900 atoms in each of two adjacent wells of a superlattice potential, formed by two standing-wave optical fields that are resonant with $\mathrm{TEM}_{00}$ modes of a Fabry-Perot optical resonator and far detuned from

\footnotetext{
${ }^{1}$ Department of Physics, University of California, Berkeley, California 94720, USA. ${ }^{2}$ Fachbereich Physik, Technische Universität Kaiserslautern, 67663 Kaiserslautern, Germany. ${ }^{3}$ Materials Sciences Division, Lawrence Berkeley National Laboratory, Berkeley, California 94720, USA.

*e-mail: spethmann@berkeley.edu
} 
a

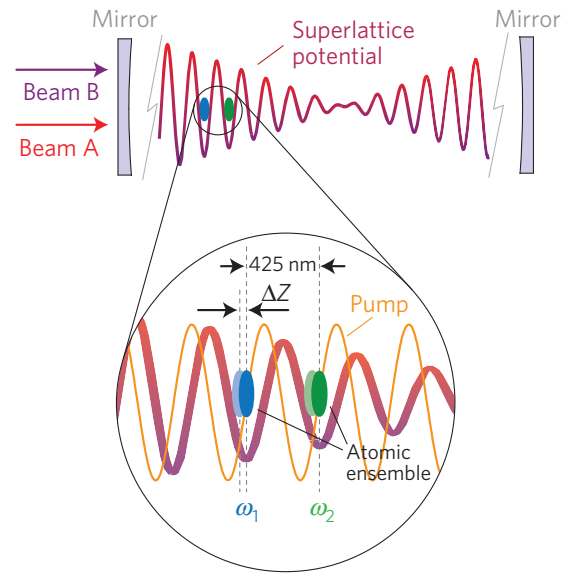

b
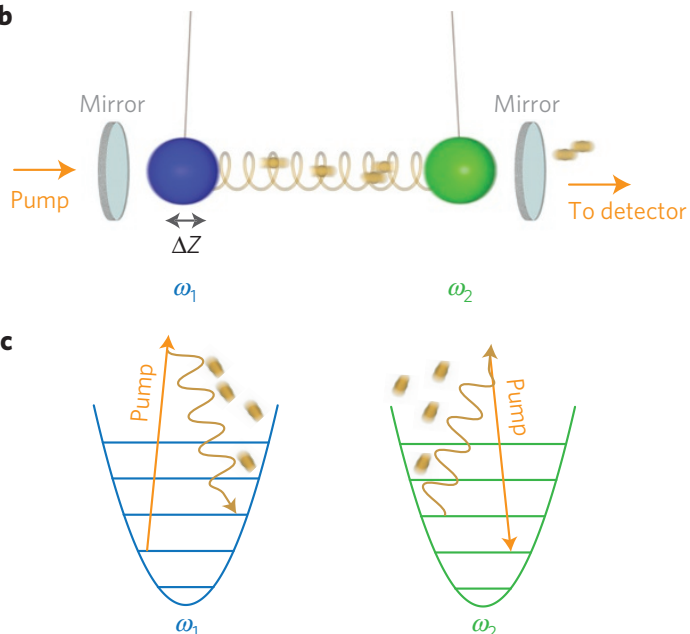

Figure 1 | Schematic set-up. a, Two addressable oscillators are created by trapping ensembles of cold atoms in two sites of a superlattice potential in a cavity. The centre-of-mass motion of each cloud of atoms is linearly coupled to the pump light, creating a system of two optomechanical oscillators.

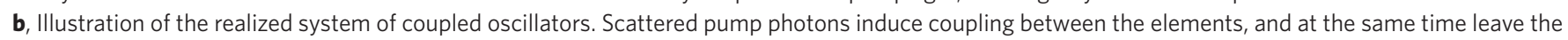
cavity, leading to back-action noise. c, In a quantized picture, cavity photons act as the force-mediating particle for the interaction. Pump light is Stokes

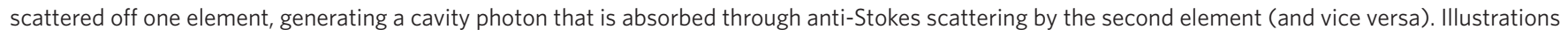
are not to scale.

atomic resonances. The deep superlattice potential prevents the exchange of atoms between the two ensembles. Optomechanical interactions are introduced by driving the cavity with light detuned by $-42 \mathrm{GHz}$ from the $\mathrm{D} 2$ atomic resonance. The driving frequency is close to another $\mathrm{TEM}_{00}$ cavity resonance, the frequency of which varies linearly with the centre-of-mass displacement of each of the trapped atomic ensembles along the cavity axis. The linear optomechanical interaction strength is quantified by the optomechanical cooperativities of the two oscillators, given as $C_{i}=4 \bar{n} g_{i}^{2} /\left(\kappa \Gamma_{i}\right)$, where $g_{i}$ is the single-photon/single-phonon coupling frequency for the $i$ th oscillator, and $\kappa=2 \pi \times 1.82 \mathrm{MHz}$ is the cavity half-linewidth. The transmission of this pump/probe light through the cavity is detected by a heterodyne optical receiver. At a typical average cavity photon number $\bar{n}=2, C_{1,2} \simeq 2$, allowing single-shot detection of the motion of each oscillator near the standard quantum limit ${ }^{19}$. Probing at this intensity reveals the resonance frequencies $\left(\left\{\omega_{1}, \omega_{2}\right\} \approx 2 \pi \times\{116.4,110\} \mathrm{kHz}\right)$ and linewidths $\left(\Gamma_{1,2} \approx 2 \pi \times 1.5 \mathrm{kHz}\right)$ of the centre-of-mass oscillations of the two ensembles. The superlattice potential allows us to tune the mechanical frequencies to be either non-degenerate (as here) or degenerate (see Methods Section 1).

The motion of the two ensembles becomes coupled when the pump light is tuned away from the cavity resonance. In the unresolved sideband regime $\left(\kappa \gg \omega_{1,2}\right)$, the optical force $F_{i}$ on oscillator $i$ varies with the oscillator positions $z_{1,2}$ as $F_{i}=-\sum_{j} k_{i, j} z_{j}$, where the optical spring constants $k_{i, j}$ are given by

$$
k_{i, j}=-\frac{\hbar g_{i} g_{j}}{Z_{\mathrm{HO}, i} Z_{\mathrm{HO}, j}} \frac{2 \bar{n} \Delta_{\mathrm{pc}}}{\kappa^{2}+\Delta_{\mathrm{pc}}^{2}}
$$

Here, $\Delta_{\mathrm{pc}}$ is the difference between the pump light and cavity resonance frequencies, $Z_{\mathrm{HO}, i}=\sqrt{\hbar / 2 M_{i} \omega_{i}}$ quantifies zero-point motion and $M_{i}$ is the total mass of the atoms in each lattice site (Methods Section 2).

Owing to these optical forces, the mechanical normal modes, describing uncoupled motion of each of the two oscillators in the absence of the optical spring, become increasingly coupled. As the optical spring gains strength, one normal mode evolves so that its position variable matches the sum of oscillator positions weighted by optomechanical coupling strengths. The optomechanical cooperativity of this 'bright' normal mode becomes collectively enhanced. The remaining 'dark' normal mode becomes increasingly decoupled from the cavity. The optical coupling also increases the frequency separation between the mechanical resonances.

We observe both these effects, the renormalization and frequency separation of the mechanical modes, by monitoring the symmetrized mechanical sideband spectrum of light transmitted from the cavity (Fig. 2). We examine the case $\Delta_{\mathrm{pc}}>0$, where a

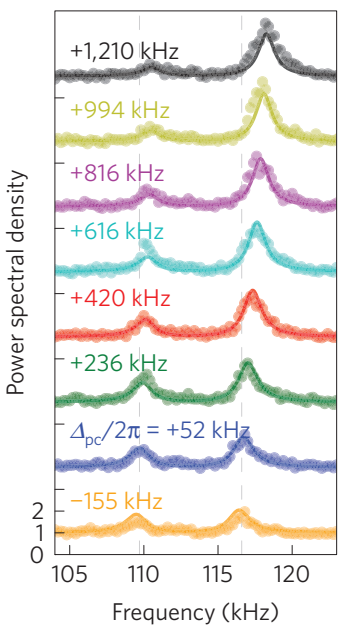

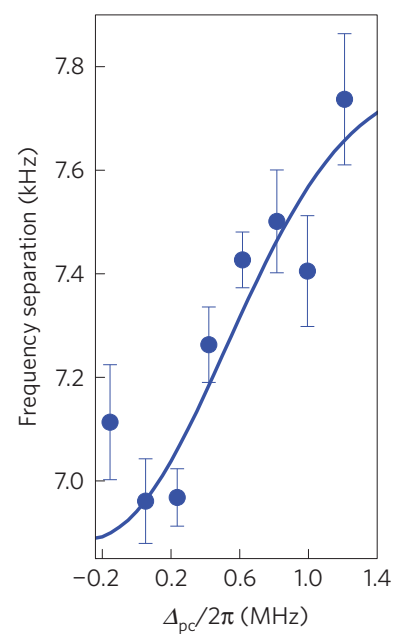

Figure $\mathbf{2}$ | Coupling of two shot-noise-driven oscillators. The cavity containing two distinct atomic ensembles is pumped with light at constant intracavity intensity ( $\bar{n} \simeq 5)$ and variable detuning $\Delta_{\mathrm{pc}}$ from cavity resonance. $\mathbf{a}$, The symmetrized sideband power spectrum of the transmitted pump light, calculated from a 5-ms-long measurement and averaged over about 250 experimental repetitions, is shown scaled to the shot noise power seen away from mechanical resonances. Spectra are offset by 3 shot noise units from one another for clarity. Optical coupling of the oscillators results in a high-cooperativity normal mode at high frequency and a low-cooperativity mode at low frequency. $\mathbf{b}$, The coupling increases the frequency separation between the modes. Bars indicate standard fitting errors. Solid lines show theoretical predictions. 

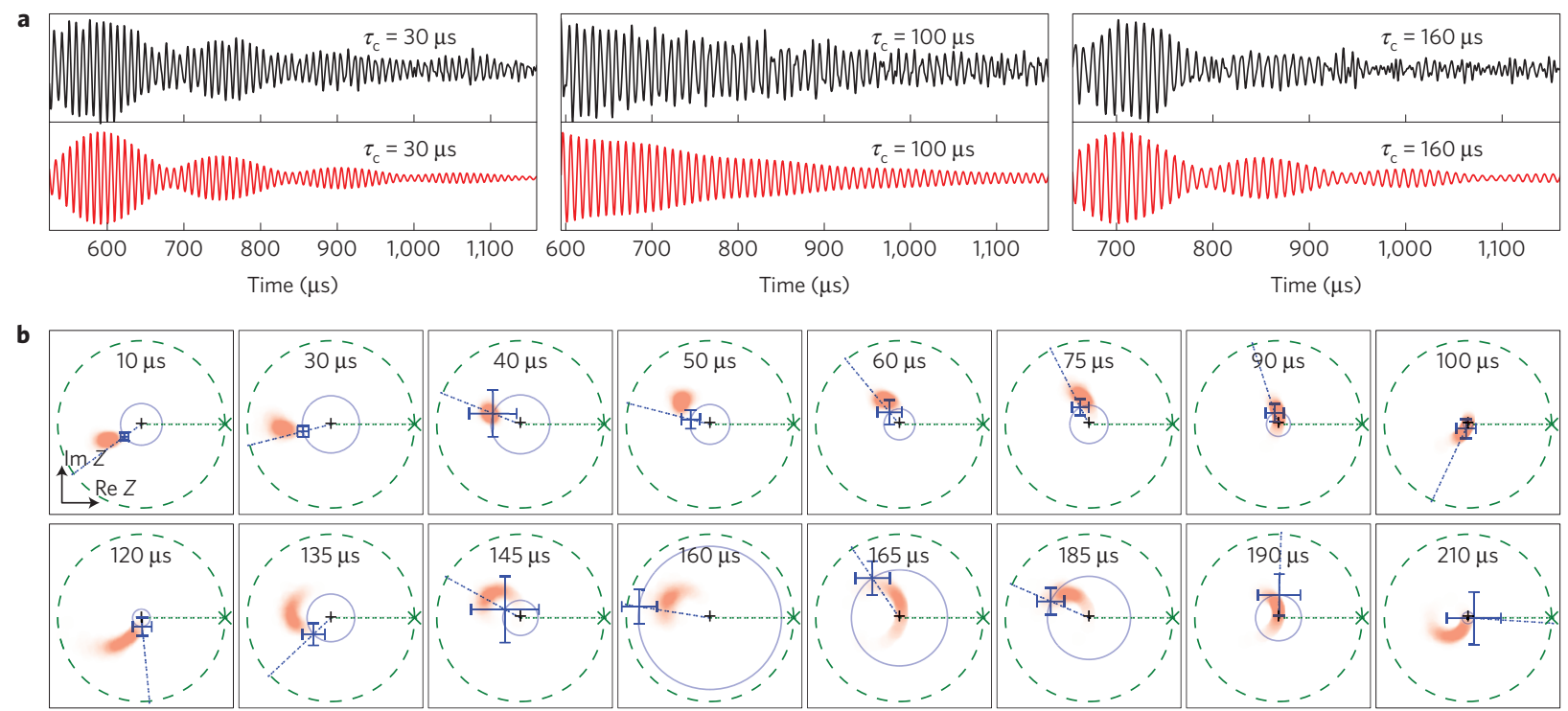

Figure $\mathbf{3}$ | Phase-coherent energy exchange. a, The optical cavity probe measures a weighted sum of the displacement of the two mechanical elements. After one element is coherently driven and an optical link between the modes is established, the beat note in the mechanical ring-down signal shows coherent oscillations of both elements. Data (black) for each $\tau_{c}$, averaged over about $10^{4}$ experimental repetitions, show reasonable agreement with zero-free-parameter numerical simulations (red). b. Phase-space plots indicating the average relative state of the high- (blue) and low-frequency (green) oscillators just after applying the optical coupling for varying times $\tau_{\mathrm{c}}$ show two cycles of energy exchange. Numerical simulations take into account experimental uncertainties (red shaded area) and show reasonable agreement. Bars denote standard statistical errors.

the optical spring leads to mechanical stiffening ${ }^{30}$. At constant intracavity photon number $(\bar{n} \simeq 5)$, we vary the pump detuning, increasing the optical spring constant up to $\left|k_{1,2}\right| \approx 1 \times 10^{-12} \mathrm{~N} \mathrm{~m}^{-1}$. We quantify the resulting coupling strength for our system by $\Omega=\left|k_{1,2}\right| /(M \bar{\omega}) \simeq 2 \pi \times 2 \mathrm{kHz}$, with the mean frequency $\bar{\omega}=\left(\omega_{1}+\omega_{2}\right) / 2$ and for simplicity we approximate $\Omega$ by employing the average mass $M$ of the two oscillators (Methods Section 3). The steady-state spectra, with the illumination time much longer than the mechanical damping time, reveal the motion of the intracavity mechanical elements driven by zero-point fluctuations, thermal noise (at a level of around $v_{\text {th }}=1.5$ phonons), and radiation-pressure quantum fluctuations.

At zero detuning, we observe two uncoupled mechanical resonances with comparable line strength. At non-zero detuning, the frequency difference between the two mechanical resonances increases. The increase is relatively small because the optical coupling in our experiment is rather weak $\left(\Omega<\omega_{1}-\omega_{2}\right)$; regenerative mechanical oscillations ${ }^{31}$ at higher optical power prevent us from increasing $\Omega$ further at steady state. The detuned pump also leads to a difference in the power of the two mechanical sidebands, with the higher-frequency resonance becoming significantly stronger. The strength difference reflects not only that the mechanical modes are renormalized so that the cavity output is more sensitive to motion of the higherfrequency normal mode, but also that this brighter mode has larger position fluctuations because it is more sensitive to radiationpressure fluctuations. A linearized model of cavity optomechanics incorporating both these compounding effects matches well to the data (see Methods Section 4).

The cavity-mediated force should allow the coherent exchange of energy between the two oscillators. To examine this exchange, we perform a form of two-dimensional spectroscopy ${ }^{32}$, consisting of three steps. First, we excite a coherent state of motion (at a level of a few $Z_{\mathrm{HO}} \simeq 0.8 \mathrm{~nm}$ ) on just the low-frequency oscillator by resonantly modulating the intensity of one of the superlattice trapping beams. During this time, the cavity is probed with onresonant light $(\bar{n}=2)$ so that the mechanical oscillators are not coupled. Second, we turn on the optical coupling between the oscillators, at the level of $\Omega \approx 2 \pi \times 4 \mathrm{kHz}$, by tuning the pump/probe light away from resonance $\left(\Delta_{\mathrm{pc}}=2 \pi \times 1.4 \mathrm{MHz}\right)$ and increasing its intensity $(\bar{n} \approx 8)$, holding the light at these settings for a variable time $\tau_{c}$. Third, we turn off the optical spring and record the phase modulation of resonant cavity probe light $(\bar{n}=2)$ to characterize the mechanical state.

Averaging the phase modulation signal over many experimental repetitions, we observe a time-domain beat note in the mechanical ring-down signal (Fig. 3a) showing that the coherent excitation is now shared by both oscillators. Applying matched data filters to this time trace, as demonstrated in ref. 33 for a one-oscillator system and detailed in Methods Section 5, we obtain measurements of both quadratures of motion of each mechanical element at the time when the optical spring is turned off (Fig. 3b). We observe a cycle time for the exchange of energy between the oscillators of about $100 \mu \mathrm{s}$. The maximal transfer of energy occurs after a halfcycle of oscillatory exchange, at the level of $A_{1}^{2} /\left(A_{1}^{2}+A_{2}^{2}\right) \approx 0.2$, where $A_{i}$ is the excitation amplitude of oscillator $i$. We compare these quadrature measurements to numerical simulations (Methods Section 5). The agreement with data is reasonable given the effects of systematic uncertainties in $\bar{n}$ and $\Delta_{\mathrm{pc}}$, and fluctuations in $M_{i}$ and $g_{i}$.

Our experiment demonstrates that two mechanical elements with no direct contact can be coupled to one another controllably through cavity optomechanics. We identify two conditions required for this controlled spring force to induce fully coherent quantum evolution of the coupled mechanical system. First, one requires the coupling rate to be larger than the thermal mechanical decoherence rate $\left(v_{\text {th }}+1\right) \Gamma$. This condition is marginally satisfied in our work. Second, one requires coherent evolution of the optical field acting as the force carrier. In our system, the non-zero cavity linewidth implies that the field leaks from the cavity, providing a means to observe the state of the optical spring. The force noise resulting from this observation limits the coherence of the mechanical system.

We observe the consequence of this force noise by applying the aforementioned matched filters to each individual repetition of the three-step experimental sequence described above (Fig. 4). Measurements before the oscillators are driven and coupled characterize their initial state. The distribution of quadrature 
a
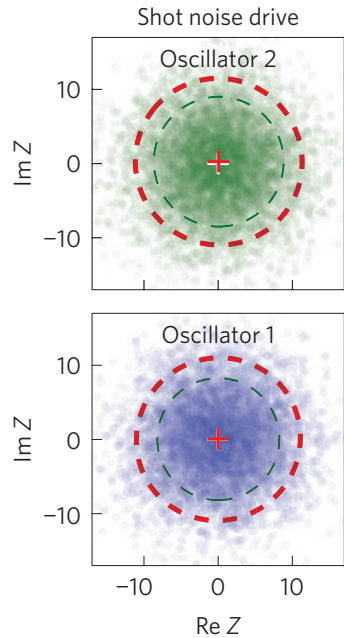
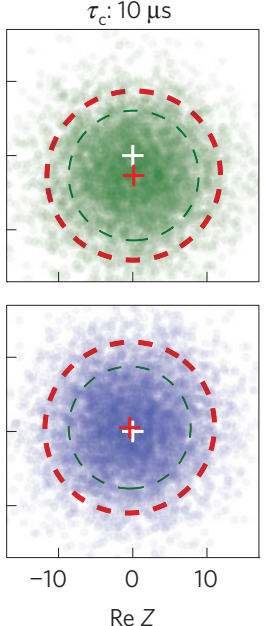

$\tau_{\mathrm{c}}: 160 \mu \mathrm{s}$
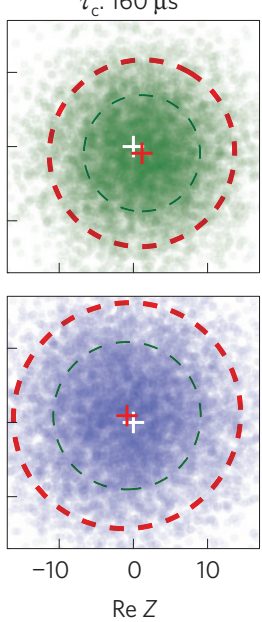

b

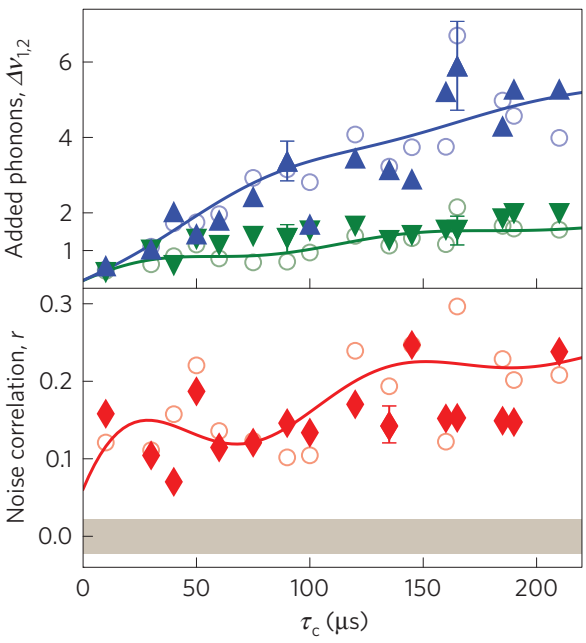

Figure 4 | Build-up of correlated back-action noise during optical coupling. a, Distributions of about $10^{4}$ quadrature measurements on the two oscillators under three conditions: no coherent excitation or coupling (left) or coherent excitation and coupling of duration $\tau_{\mathrm{c}}$ (centre and right). Dashed lines indicate $1-\sigma$ variance ellipses for signal (red) and optical shot noise (black); the excess signal variance quantifies the incoherent excitation. b, Top: optical coupling increases the incoherent excitation level, plotted as the number of added phonons for the high- (blue triangle) and low-frequency (green downtriangle) oscillator. The measured data show agreement with zero-free-parameter theory calculated for each $\tau_{\mathrm{c}}$ (open symbols); see Methods Section 5 . Bottom: the coupling leads to correlations in the displacements of the two oscillators, quantified by the covariance $r$ (red lozenge) between their quadratures. Correlations show the expected trend, slightly reduced below zero-free-parameter expectations (open symbols). The measured 1- $\sigma$ correlations for uncoupled oscillators are indicated by the grey shaded range. Solid lines indicate theory for average experimental parameters. Bars show typical 1- $\sigma$ standard statistical errors; a plot with all error bars can be found in the Supplementary Information.

measurements is centred about zero. The excess variance in the quadrature measurements above the optical shot noise level reflects the incoherent initial excitation of both oscillators at the level of 2.5 phonons. After the oscillators are coherently excited and optically coupled, the quadrature measurement distributions are offset from the origin, reflecting the coherent motion of both elements. In addition, the variance of these measurements increases, showing that back-action noise during the application of the optical spring incoherently excites both oscillators. The incoherent excitation strength is different for the two oscillators, with the high-frequency oscillator gaining energy faster than the low-frequency oscillator. This difference occurs because the bright coupled mechanical mode is more strongly disturbed by back-action, and, in the weak-spring regime explored in this work, this bright mode corresponds to larger displacement of the high-frequency oscillator than of the low-frequency oscillator. Our observations are in good agreement with expectations from zero-free-parameter theory (see Methods Section 5).

Interestingly, the back-action noise results in correlated fluctuations of the two oscillators. These correlations, quantified by examining the covariance between motional quadratures of the two oscillators, grow in strength during the application of the optical spring. The correlations indicate that, when coupled, the two mechanical oscillators are coupled to the same bath of quantum optical force fluctuations.

Fluctuations of the optical spring force are found to add one phonon of incoherent excitation within about $30 \mu \mathrm{s}$, a time shorter than the coherent coupling time $(\Omega / 2 \pi)^{-1} \simeq 250 \mu \mathrm{s}$. This comparison implies that for the conditions of our experimentpump light detuned about one half-linewidth from the cavity resonance-the force linking the two mechanical oscillators cannot be used to exchange non-classical states of motion, such as phonon Fock states, between the oscillators. However, this limitation is not fundamental, and may be mitigated by driving the cavity further from its resonance frequency ${ }^{8}$ - an effect similar to the avoidance of spontaneous emission on a far-detuned Raman transition-or through post-selection or feedback using light exiting the cavity.
Our results provide direct insight into the limits that back-action imposes on the coupling of quantum systems with real photons. This improved understanding might help to overcome or exploit the effects of quantum back-action in complex multi-element quantum systems ${ }^{14,34}$.

\section{Methods}

Methods and any associated references are available in the online version of the paper.

Received 30 January 2015; accepted 15 September 2015; published online 19 October 2015

\section{References}

1. Brown, K. R. et al. Coupled quantized mechanical oscillators. Nature 471, 196-199 (2011).

2. Harlander, M., Lechner, R., Brownnutt, M., Blatt, R. \& Hänsel, W. Trapped-ion antennae for the transmission of quantum information. Nature 471, 200-203 (2011)

3. Pashkin, Y. A. et al. Quantum oscillations in two coupled charge qubits. Nature 421, 823-826 (2003)

4. Julsgaard, B., Kozhekin, A. \& Polzik, E. S. Experimental long-lived entanglement of two macroscopic objects. Nature 413, 400-403 (2001).

5. Ritter, S. et al. An elementary quantum network of single atoms in optical cavities. Nature 484, 195-200 (2012).

6. Hofmann, J. et al. Heralded entanglement between widely separated atoms. Science 337, 72-75 (2012)

7. Pellizzari, T., Gardiner, S. A., Cirac, J. I. \& Zoller, P. Decoherence, continuous observation, and quantum computing: A cavity QED model. Phys. Rev. Lett. 75, 3788-3791 (1995).

8. Buchmann, L. F. \& Stamper-Kurn, D. M. The quantum/classical transition in mediated interactions. Ann. Phys. 527, 156-161 (2015).

9. Woolley, M. J. \& Clerk, A. A. Two-mode back-action-evading measurements in cavity optomechanics. Phys. Rev. A 87, 063846 (2013).

10. Hartmann, M. J. \& Plenio, M. B. Steady state entanglement in the mechanical vibrations of two dielectric membranes. Phys. Rev. Lett. 101, 200503 (2008).

11. Bhattacharya, M. \& Meystre, P. Multiple membrane cavity optomechanics. Phys. Rev. A 78, 041801 (2008)

12. Seok, H., Buchmann, L. F., Wright, E. M. \& Meystre, P. Multimode strong-coupling quantum optomechanics. Phys. Rev. A 88, 063850 (2013). 
13. Verlot, P., Tavernarakis, A., Briant, T., Cohadon, P.-F. \& Heidmann, A. Scheme to probe optomechanical correlations between two optical beams down to the quantum level. Phys. Rev. Lett. 102, 103601 (2009).

14. Stannigel, K. et al. Optomechanical quantum information processing with photons and phonons. Phys. Rev. Lett. 109, 013603 (2012).

15. Aspelmeyer, M., Kippenberg, T. J. \& Marquardt, F. Cavity optomechanics. Rev. Mod. Phys. 86, 1391-1452 (2014).

16. Brooks, D. W. C. et al. Non-classical light generated by quantum-noise-driven cavity optomechanics. Nature 488, 476-480 (2012).

17. Purdy, T. P., Yu, P.-L., Peterson, R. W., Kampel, N. S. \& Regal, C. A. Strong optomechanical squeezing of light. Phys. Rev. X 3, 031012 (2013).

18. Safavi-Naeini, A. H. et al. Squeezed light from a silicon micromechanical resonator. Nature 500, 185-189 (2013).

19. Schreppler, S. et al. Optically measuring force near the standard quantum limit. Science 344, 1486-1489 (2014).

20. Suh, J. et al. Mechanically detecting and avoiding the quantum fluctuations of a microwave field. Science 344, 1262-1265 (2014).

21. Palomaki, T. A., Teufel, J. D., Simmonds, R. W. \& Lehnert, K. W. Entangling mechanical motion with microwave fields. Science 342, 710-713 (2013).

22. Lin, Q. et al. Coherent mixing of mechanical excitations in nano-optomechanical structures. Nature Photon. 4, 236-242 (2010).

23. Massel, F. et al. Multimode circuit optomechanics near the quantum limit. Nature Commun. 3, 987 (2012).

24. Shkarin, A. et al. Optically mediated hybridization between two mechanical modes. Phys. Rev. Lett. 112, 013602 (2014).

25. Zhang, M. et al. Synchronization of micromechanical oscillators using light. Phys. Rev. Lett. 109, 233906 (2012).

26. Black, A. T., Chan, H. W. \& Vuletić, V. Observation of collective friction forces due to spatial self-organization of atoms: From rayleigh to bragg scattering. Phys. Rev. Lett. 91, 203001 (2003).

27. Baumann, K., Guerlin, C., Brennecke, F. \& Esslinger, T. Dicke quantum phase transition with a superfluid gas in an optical cavity. Nature 464, 1301-1306 (2010).

28. Carmichael, H. J. Quantum trajectory theory for cascaded open systems. Phys. Rev. Lett. 70, 2273-2276 (1993).
29. Botter, T., Brooks, D. W. C., Schreppler, S., Brahms, N. \& Stamper-Kurn, D. M. Optical readout of the quantum collective motion of an array of atomic ensembles. Phys. Rev. Lett. 110, 153001 (2013).

30. Corbitt, T. et al. An all-optical trap for a gram-scale mirror. Phys. Rev. Lett. 98, 150802 (2007)

31. Kippenberg, T. J., Rokhsari, H., Carmon, T., Scherer, A. \& Vahala, K. J. Analysis of radiation-pressure induced mechanical oscillation of an optical microcavity. Phys. Rev. Lett. 95, 033901 (2005).

32. Ernst, R. R., Bodenhausen, G. \& Wokaun, A. Principles of Nuclear Magnetic Resonance in One and Two Dimensions (Clarendon, 1987).

33. Palomaki, T. A., Harlow, J. W., Teufel, J. D., Simmonds, R. W. \& Lehnert, K. W. Coherent state transfer between itinerant microwave fields and a mechanical oscillator. Nature 495, 210-214 (2013).

34. Joshi, C., Akram, U. \& Milburn, G. J. An all-optical feedback assisted steady state of an optomechanical array. New J. Phys. 16, 023009 (2014).

\section{Acknowledgements}

This work was supported by the Air Force Office of Scientific Research and NSF. N.S. was supported by a Marie Curie International Outgoing Fellowship, J.K. and S.S. by the US

Department of Defense through the National Defense Science and Engineering Graduate Fellowship Program, and L.B. by the Swiss National Science Foundation.

\section{Author contributions}

Experimental data were taken by N.S., J.K. and S.S.; L.B. developed the theoretical model. All authors were involved with experimental design, data analysis, and production of the manuscript.

\section{Additional information}

Supplementary information is available in the online version of the paper. Reprints and permissions information is available online at www.nature.com/reprints. Correspondence and requests for materials should be addressed to N.S.

\section{Competing financial interests}

The authors declare no competing financial interests. 


\section{Methods}

1. A gas of cold atoms as optomechanical oscillator. We create a system where the collective axial centre-of-mass motion of a trapped atomic cloud is linearly coupled to the intracavity pump light, whereas all other axial modes average out and are not measured. This system is equivalent to other realizations of cavity

optomechanics $^{35,36}$, which is reflected in a common theoretical description ${ }^{37}$.

Preparation of mechanical oscillators. We load a sample of ultracold ${ }^{87} \mathrm{Rb}$ atoms into two wells of a superlattice potential, created by exciting two TEM $\mathrm{T}_{00}$ modes of the cavity, one at $\lambda_{\mathrm{b}}=862 \mathrm{~nm}$, and a second at $\lambda_{\mathrm{a}}=843 \mathrm{~nm}$ (illustrated in Fig. 1). In this way, we prepare two oscillators with a different mechanical frequency. To control the oscillator frequencies, the intensities of both lattice beams are detected after transmission through the cavity and actively stabilized. Drifts in the detection efficiency cause the centre frequencies of both oscillators to vary slowly over time. We directly stabilize the low-frequency oscillator to $\omega_{2}=2 \pi \times 110 \mathrm{kHz}$, at $\Delta_{\mathrm{pc}} \approx 0, \bar{n} \approx 2$, by extracting the mechanical sideband frequency on the cavity probe light over several experimental repetitions and feeding back to the intensity of one of the superlattice beams. The lock limits the drift of the high-frequency oscillator $\left(\omega_{1}=2 \pi \times 116.4 \mathrm{kHz}\right)$ to about $\pm 200 \mathrm{~Hz}$. The thermal phonon occupation of the mechanical oscillators in the superlattice (with the probe light off) is calibrated by employing time-of-flight measurements of the atomic cloud to $v_{\text {th }} \approx 1.5$ phonons in the relevant axial direction of the lattice.

Optomechanical interaction. The atoms are loaded into optical lattice sites centred at locations where the probe light intensity has its highest gradient. We thereby create two oscillators with comparable (nearly maximal) linear optomechanical interaction strength. The non-zero curvature of the probe light causes residual quadratic coupling measured to be $2 \pi \times-40(+208) \mathrm{Hz} /$ photon for the low-frequency (high-frequency) oscillator ${ }^{35}$.

The parameters of the mechanical modes (resonance frequencies $\omega_{i}$ and damping rates $\Gamma_{i}$ ) can be extracted from fits to the sidebands in the phase quadrature of the optical heterodyne detection when probed on cavity resonance. The optomechanical cooperativity $C_{i}$ can be measured from the peak height of the incoherent response $P_{i}$ via

$$
C_{i}=-\left(v_{\mathrm{th}, i}+1 / 2\right)+\sqrt{\left(v_{\mathrm{th}, i}+1 / 2\right)^{2}+\frac{2 P_{i}\left(\omega_{i}\right) / S_{\mathrm{SN}}-1}{4 \epsilon}}
$$

where $S_{\mathrm{SN}}$ is the total spectral density of the shot noise power and $\epsilon \approx 0.05$ is half the quantum efficiency (use of heterodyne rather than homodyne detection) ${ }^{19}$. The single-photon/single-phonon coupling strength $g_{i}$ for each oscillator is then determined by $C_{i}=4 \bar{n} g_{i}^{2} /\left(\kappa \Gamma_{i}\right)$, with the mean intracavity photon number $\bar{n}$. In this work, we typically prepare samples with $g_{i} \approx 2 \pi \times 24 \mathrm{kHz}$.

Coherent excitation of a single oscillator. To coherently excite only the low-frequency oscillator, we apply resonant intensity modulation to one of the superlattice beams with a pulse shape corresponding to a truncated Blackman envelope $^{38}$. The resulting narrow spectral width ensures residual amplitude excitation of the high-frequency oscillator on the order of $10^{-2}$ (see Supplementary Fig. 1).

2. Optical forces and induced optical spring. In the unresolved sideband regime $\left(\kappa \gg \omega_{1,2}\right)$, the optomechanical interaction of the cavity light with the oscillators can be described by an optical dipole force acting on oscillator $i$ which is linear in intracavity photon number $\bar{n}$ :

$$
F_{i}=-\frac{\hbar g_{i}}{Z_{\mathrm{HO}, i}} \bar{n}
$$

with $Z_{\mathrm{HO}, i}$ the harmonic oscillator length. A displacement $z_{j}$ of an oscillator from its equilibrium position changes the cavity resonance frequency by $g_{j} z_{j} / Z_{\mathrm{HO}, j}$. In a cavity driven with a constant frequency pump, this displacement translates to first order into a change of the intracavity photon number $\bar{n}$ by

$$
\delta \bar{n} \simeq-\bar{n} \frac{2 \Delta_{\mathrm{pc}}}{\kappa^{2}+\Delta_{\mathrm{pc}}^{2}} \frac{g_{j}}{Z_{\mathrm{HO}, j}} z_{j}
$$

which varies the force on oscillator $i$ by the amount

$$
F_{i}=\sum_{j} \frac{\hbar g_{j} g_{i}}{Z_{\mathrm{HO}, i} Z_{\mathrm{HO}, j}} \frac{2 \bar{n} \Delta_{\mathrm{pc}}}{\kappa^{2}+\Delta_{\mathrm{pc}}^{2}} z_{j}=-k_{i, j} z_{j}
$$

Here, we have dropped constant terms and introduced the spring constant of equation (1):

$$
k_{i, j}=-\frac{\hbar g_{i} g_{j}}{Z_{\mathrm{HO}, i} Z_{\mathrm{HO}, j}} \frac{2 \bar{n} \Delta_{\mathrm{pc}}}{\kappa^{2}+\Delta_{\mathrm{pc}}^{2}}
$$

Coupling pulses. To prevent additional excitation of the mechanical modes from rapid changes in intracavity photon number, when turning the optical spring on and off, we vary the probe/pump settings gradually over a $20 \mu$ s switching time. We repeat excitation and coupling pulses several times within each run of the experiment to facilitate data taking. After applying a pulse, the mechanical system is allowed to re-equilibrate under constant illumination (at $\bar{n} \simeq 2$ and $\Delta_{\mathrm{pc}} \simeq 0$ ) before the spectroscopy sequence is repeated after $2 \mathrm{~ms}$. After five repetitions, the atoms are released from their trap and a new atomic gas is cooled and placed within the cavity.

Choosing a sufficiently slow repetition rate allows one to read out different parts of the time trace: the first time window covers the ring-down following the excitation and coupling. The second time window is shifted by several ring-down times, so that coherent and incoherent excitation has damped out and each oscillator achieves a steady-state incoherent phonon occupation from mechanical damping and the heating from radiation pressure fluctuations. (Fig. 4a). The temperature of both oscillators in this time window corresponds to the initial incoherent excitation and is measured by sideband asymmetry ${ }^{36}$ for each $\tau_{\mathrm{c}}$; its value is typically 2.5 phonons. With an optomechanical cooperativity of $C_{1,2} \approx 2$, corresponding to about one phonon due to back-action ${ }^{36}$, and a thermal base occupation of $v_{\text {th }} \approx 1.5$ phonons (see above), we find good agreement with expectations.

3. Simplified classical model. It is illustrative to quantify the coupling strength $\Omega$ in a simplified system. For equal spring constants $k=k_{i, j}$ and masses $M=M_{1,2}$, but different natural frequencies $\omega_{i}$, the time evolution equations for displacements of two coupled oscillators are

$$
-\left(\begin{array}{l}
\ddot{z}_{1} \\
\ddot{z}_{2}
\end{array}\right)=\left(\begin{array}{cc}
(\bar{\omega}+\delta / 2)^{2}+k / M & k / M \\
k / M & (\bar{\omega}-\delta / 2)^{2}+k / M
\end{array}\right)\left(\begin{array}{l}
z_{1} \\
z_{2}
\end{array}\right)
$$

with the mean frequency $\bar{\omega}=\left(\omega_{1}+\omega_{2}\right) / 2$ and the frequency splitting $\delta=\omega_{1}-\omega_{2}$. Normal mode frequencies of the coupled oscillators are given by the eigenvalues of this matrix:

$$
\omega_{ \pm}^{2}=\delta^{2} / 4+k / M+\bar{\omega}^{2} \pm \sqrt{(k / M)^{2}+\delta^{2} \bar{\omega}^{2}}
$$

The system is in the weak (strong) coupling regime, if the splitting due to coupling is small (large) compared to $|\delta|$. Correspondingly we quantify the strength of the coupling by $\Omega=k /(M \bar{\omega})$. In this work, we typically operate at $\Omega \approx 2 \pi \times(2-4) \mathrm{kHz}<\delta=2 \pi \times 6.4 \mathrm{kHz}$.

The oscillator properties for the entire data set used vary by less than $15 \%$, which justifies the use of the simplified coupling strength $\Omega$ in the discussion of general features of our system. Calculations performed to predict the experimental observations take variations of $g_{i}, M_{i}$ and $\Gamma_{i}$ into account (see below).

4. Linear amplifier model extension to two oscillators. We generalize the treatment of linearized cavity optomechanics with a single mechanical mode in refs 36,37 with the following definitions:

$$
\begin{gathered}
\left(\begin{array}{c}
\hat{\tilde{a}}_{+} \\
\hat{\tilde{a}}_{-}
\end{array}\right)=\mathbf{F}_{\mathbf{a}}\left[\sum_{j=1}^{N} \mathbf{T}_{\mathbf{j}}\left(\begin{array}{c}
\hat{\tilde{z}}_{j} \\
\tilde{\tilde{p}}_{j}
\end{array}\right)+\sqrt{2 \kappa}\left(\begin{array}{c}
\hat{\tilde{\alpha}}_{\mathrm{in}+} \\
\tilde{\tilde{\alpha}}_{\mathrm{in}-}
\end{array}\right)\right] \\
\left(\begin{array}{c}
\hat{\tilde{z}}_{j} \\
\tilde{\tilde{p}}_{j}
\end{array}\right)=\mathbf{F}_{\mathbf{b} \mathbf{j},}\left[\mathbf{T}_{\mathbf{j}}\left(\begin{array}{c}
\hat{\tilde{a}}_{+} \\
\hat{\tilde{a}}_{-}
\end{array}\right)+\sqrt{\boldsymbol{\Gamma}_{\mathbf{j}}}\left(\begin{array}{c}
\hat{\tilde{\eta}}_{\text {in }+, j} \\
\tilde{\tilde{\eta}}_{\mathrm{in}-, j}
\end{array}\right)\right]
\end{gathered}
$$

where $\mathbf{F}_{\mathbf{a}}$ is the response matrix for the optical cavity, $\mathbf{F}_{\mathbf{b}, \mathbf{j}}$ the matrix corresponding to the mechanical response of oscillator $j$ and $\mathbf{T}_{\mathbf{j}}$ the matrix capturing the coupling of oscillator $j$ to the light field of the cavity. Photonic and phononic inputs (including noise) are symbolized by $\hat{\tilde{\alpha}}_{\text {in }+}, \hat{\tilde{\alpha}}_{\text {in- }}$ and $\hat{\tilde{\eta}}_{\text {in }+, j}, \hat{\tilde{\eta}}_{\text {in-,j, }}$, respectively.

5. Data analysis and numerical simulations. The lab-frame master equation for the density operator $\rho$ describing the cavity field (field operator $\hat{a}$ ) and the mechanical modes including optical pump and dissipation is given by

$$
\begin{aligned}
\frac{\mathrm{d} \rho}{\mathrm{d} t}= & \frac{-i}{\hbar}\left[\mathcal{H}_{m}+\mathcal{H}_{o}+\mathcal{H}_{\mathrm{OM}}, \rho\right]+\kappa \mathcal{L}(\hat{a}) \rho \\
& +\sum_{j=1}^{2}\left[\Gamma_{j}\left(v_{\mathrm{th}, j}+1\right) \mathcal{L}\left(\hat{b}_{j}\right) \rho+\Gamma_{j} v_{\mathrm{th}, j} \mathcal{L}\left(\hat{b}_{j}^{\dagger}\right) \rho\right]
\end{aligned}
$$

with

$$
\begin{gathered}
\mathcal{H}_{m}=\sum_{j=1}^{2} \hbar \omega_{j} \hat{b}_{j}^{\dagger} \hat{b}_{j} \\
\mathcal{H}_{o}=\hbar \omega_{\mathrm{c}} \hat{a}^{\dagger} \hat{a}+\eta \hat{a}^{\dagger} \mathrm{e}^{-i \omega_{\mathrm{L}} t}+\eta^{*} \hat{a} \mathrm{e}^{i \omega_{\mathrm{L}} t} \\
\mathcal{H}_{\mathrm{OM}}=\sum_{j=1}^{2} \hbar g_{j} \hat{a}^{\dagger} \hat{a}\left(\hat{b}_{j}+\hat{b}_{j}^{\dagger}\right)
\end{gathered}
$$


with the cavity resonance frequency $\omega_{\mathrm{c}}$, the optical pumping rate $\eta$ and frequency $\omega_{\mathrm{L}}$, the mechanical (optical) dissipation rates $\Gamma_{j}(\kappa)$ as well as the mechanical reservoir occupation numbers $v_{\mathrm{th}, j}$. The Lindblad superoperator $\mathcal{L}$ is given by

$$
\mathcal{L}(\hat{O}) \rho=\hat{O} \rho \hat{O}^{\dagger}-\frac{1}{2}\left(\hat{O}^{\dagger} \hat{O} \rho+\rho \hat{O}^{\dagger} \hat{O}\right)
$$

where $\hat{O}$ is one of the operators $\hat{a}, \hat{b}_{j}$.

The coupling of each oscillator to its independent, Markovian bath is captured by the third term of the master equation (10). Phonon annihilation and creation operators $\hat{b}_{j}$ are related to the displacement operator by

$$
\hat{z}_{i}=\sqrt{\frac{M_{i} \omega_{i}}{2 \hbar}}\left(\hat{b}_{i}+\hat{b}_{i}^{\dagger}\right)
$$

and the dimensionless, complex phasor $Z$ used in the main paper is identified with $(1 / \sqrt{2})\langle\hat{b}\rangle$ for each oscillator. Moving to a co-rotating frame for the mechanical modes, $\hat{b}_{i} \rightarrow \mathrm{e}^{\mathrm{i} \omega_{i} t} \hat{b}_{i}$, linearizing the interaction and eliminating the cavity field yields the master equation for the reduced density operator $\rho_{\mathrm{m}}$. Introducing $\hat{\mathbf{b}}^{\top}=\left(\hat{b}_{1}, \hat{b}_{2}, \hat{b}_{1}^{\dagger}, \hat{b}_{2}^{\dagger}\right)$ it reads

$$
\frac{\mathrm{d} \rho_{\mathrm{m}}}{\mathrm{d} t}=-\frac{i}{\hbar}\left[\mathcal{H}, \rho_{\mathrm{m}}\right]+\sum_{i, j=1}^{4} h_{i j}\left(\hat{\mathbf{b}}_{i} \rho \hat{\mathbf{b}}_{j}^{\dagger}-\frac{1}{2}\left(\rho \hat{\mathbf{b}}_{j}^{\dagger} \hat{\mathbf{b}}_{i}+\hat{\mathbf{b}}_{j}^{\dagger} \hat{\mathbf{b}}_{j} \rho\right)\right)
$$

with the effective Hamiltonian

$$
\mathcal{H}=\delta \Omega_{1} \hat{b}_{1}^{\dagger} \hat{b}_{1}+\delta \Omega_{2} \hat{b}_{2}^{\dagger} \hat{b}_{2}+J\left(\hat{b}_{1} \hat{b}_{2}^{\dagger} \mathrm{e}^{-i\left(\omega_{1}-\omega_{2}\right) t}+\hat{b}_{1}^{\dagger} \hat{b}_{2} \mathrm{e}^{i\left(\omega_{1}-\omega_{2}\right) t}\right)
$$

Details of the calculation along with the explicit form of frequency shifts $\delta \Omega_{i}$ and coupling strength $J$ can be found in ref. 39. In the unresolved sideband limit we recover the optical springs of Methods Sections 2 and 3.

Matched filter. The matched filter corresponds to a least squares fit of the data to a sum of two (complex) template functions, that is, $\sum_{j=\{1,2\}} \alpha_{j} \mathrm{e}^{i \omega_{j} t-\Gamma_{j} t}$, where we extract $\Gamma_{1,2}$ and $\omega_{1,2}$ from measurements on shot-noise-driven oscillators with probe light resonant with the cavity (as in Fig. 2a). We correct the filter output $\alpha_{j}$ for slight non-orthogonality of the template functions. The real and imaginary parts of $\alpha_{j}$ represent the best estimate of the state of oscillator $j$ in its phase space at $\tau_{\mathrm{c}}$. A direct comparison between the distribution of filter coefficients and fits to time traces is given in Supplementary Fig. 1.

We scale the real and imaginary parts of the filter output to each harmonic oscillators' units by multiplying with ${ }^{19} 1 / \sqrt{\epsilon S_{\mathrm{SN}} C_{i} \Gamma_{i}}$. This takes variations in $\Gamma_{i}$ and $C_{i}$ between oscillators into account and allows identification of filter coefficients with dimensionless harmonic oscillator displacements $Z_{i}$.

Simulation of coherent transfer in the time domain. We obtain the theoretical coherent motion of the coupled oscillators by integrating the equations of motion, with specific parameters $\left\{g_{j}, \bar{n}, \omega_{j}, \Delta_{\mathrm{pc}}, v_{\mathrm{th}, j}, \Gamma_{j}, M_{j}\right\}$ for each $\tau_{\mathrm{c}}$ data set and for each oscillator determined as described above. To account for experimental imperfections in the created coupling pulses (slightly varying transients), we feed the averaged, measured waveforms for $\bar{n}$ and $\Delta_{\mathrm{pc}}$ for each $\tau_{\mathrm{c}}$ data set into the calculation and numerically obtain the time-domain evolution of both oscillators shown in Fig. 3.

To estimate the uncertainty of the predicted evolution, we vary the experimental parameters according to their uncertainty by randomly choosing values from a corresponding Gaussian distribution. Repeating the simulation for a few hundred sets of random samples gives a red shaded uncertainty in Fig. 3.

Shot-to-shot fluctuations of the loading position and atom number in each oscillator decreases the coupling strength and the observed coherent transfer. However, the single-shot resolution of our experiment does not allow a quantitative estimation of this effect and the agreement with expectation suggests this effect to be small.

Coherent energy exchange rate. The effective frequency splitting between the two oscillators, determining the coherent energy exchange rate, is increased by two effects during the coupling. The coupling itself increases the natural frequency splitting of $\delta / 2 \pi \simeq 6.4 \mathrm{kHz}$ by an amount of about $1.5 \mathrm{kHz}$, while the quadratic optomechanical coupling to the probe adds another $1.5 \mathrm{kHz}$ difference that depends only on the probe light intensity and not on its frequency. We expect a cycle time for coherent exchange of energy of around $(9.4 \mathrm{kHz})^{-1}$ and observe reasonable agreement (Fig. 3).

Dissipation and decoherence. Empirically, we observe the decay of the mechanical states of the two oscillators by three measurements: incoherent measurement of the the spectral power density of phase modulations of the cavity probe light under steady-state conditions (as in Fig. 2); phase-coherent mechanical ring-down measurements after the oscillators are excited coherently (see Supplementary Fig. 1 and Fig. 3); and incoherent measurements of the decay of the total energy in the two oscillators after they have been heated by back-action noise during coupling (see Fig. 4). The first two methods are sensitive to both energy relaxation and dephasing processes, whereas the last method measures predominantly energy relaxation. We find the decay rates in all three measurements agree (see also discussion of data filters for ring-down measurements), implying that energy relaxation is the dominant decoherence mechanism.

Furthermore, we expect that the mechanical heat baths for the two spatially separated mechanical oscillators should be independent. Damping with a common heat bath would have resulted in correlated fluctuations of the two oscillators. Through data such as in Fig. 4b, taken in the absence of the optical spring, we find no evidence for such correlations.

Accordingly, we choose a decoherence model with each oscillator independently coupled to a Markovian finite-temperature heatbath, with a rate experimentally determined as described above.

The matrix $h$ in equation (16) describes the non-unitary evolution of the mechanical degrees of freedom, including independent heatbaths and noise due to the coupling to the cavity field:

$$
h=\left(\begin{array}{cccc}
\Gamma_{-}^{(1)}+\Gamma_{1}\left(v_{\mathrm{th}, 1}+1\right) & \Gamma_{-} & 0 & 0 \\
\Gamma_{-} & \Gamma_{-}^{(2)}+\Gamma_{2}\left(v_{\mathrm{th}, 2}+1\right) & 0 & 0 \\
0 & 0 & \Gamma_{+}^{(1)}+\Gamma_{1} v_{\mathrm{th}, 1} & \Gamma_{+} \\
0 & 0 & \Gamma_{+} & \Gamma_{+}^{(2)}+\Gamma_{2} v_{\mathrm{th}, 2}
\end{array}\right)
$$

In the unresolved sideband regime we have

$$
\begin{gathered}
\Gamma_{ \pm}^{(j)}=\frac{2 g_{j}^{2} \bar{n} \kappa}{\kappa^{2}+\left(-\Delta_{\mathrm{pc}} \pm \omega_{j}\right)^{2}} \\
\Gamma_{ \pm}=\frac{\Gamma_{ \pm}^{(1)}+\Gamma_{ \pm}^{(2)}}{2}
\end{gathered}
$$

Open symbols in Fig. 4 result from numerically integrating the master equation (16) for the second-order expectation values for independen experimental parameters $\left\{g_{j}, \bar{n}, \omega_{j}, \Delta_{p c}, v_{\mathrm{th}, j}, \Gamma_{j}\right\}$ for each $\tau_{c}$, whereas the lines are results of employing the experimental parameters averaged over all $\tau_{\mathrm{c}}$ and including the ramps to establish and turn off the optical spring.

Calculation of added phonons. The phase-space distribution measured by the matched filters is employed to determine the number of added phonons. Error ellipses give the phase-space areas $A_{\text {ref }}$ for shot-noise-driven oscillators and $A_{\mathrm{rd}}$ after excitation and coupling, the contribution of shot noise is accounted for by calculating the output of the filter off mechanical resonance (black error ellipses in Fig. 4). The number of added phonons (Fig. $4 \mathrm{~b}$, top panel) is then given by $\Delta v_{1,2}=\left(\left(A_{\text {rd }}-A_{\text {ref }}\right) / A_{\text {ref }}\right)\left(P_{+} /\left(P_{-}-P_{+}\right)\right)$, where $P_{+}\left(P_{-}\right)$is the power of the blue (red) sideband. We estimate the standard statistical errors by error propagation from uncertainties in sideband power and phase-space areas. We show typical uncertainties in Fig. 4 and plot the full results in Supplementary Fig. 2a.

We test the statistical agreement between theory and experiment by calculating $\chi_{\text {red }}^{2}=(1 / N-1) \sum_{N}\left(\left(v_{\exp }-v_{\text {theo }}\right)^{2} / \sigma^{2}\right)=0.97$, where $N$ is the number of observations. The near-unity result indicates a good statistical agreement between theory and experiment.

Noise correlations. Noise correlations are quantified by

$$
r_{\mathrm{Re}}=\frac{\left\langle\operatorname{Re}\left(\hat{Z}_{1}\right) \operatorname{Re}\left(\hat{Z}_{2}\right)\right\rangle}{\sqrt{\left\langle\operatorname{Re}\left(\hat{Z}_{1}\right)^{2}\right\rangle\left\langle\operatorname{Re}\left(\hat{Z}_{2}\right)^{2}\right\rangle}}
$$

and similarly for the orthogonal quadrature $\operatorname{Im}\left(\hat{Z}_{i}\right)$. For our system and conditions equation (16) predicts non-zero correlations $r_{\mathrm{Re}}=r_{\mathrm{Im}} \equiv r$. We treat the correlations between real and imaginary parts of the coefficients as independent measurements of the correlation $r$. To obtain correlations from the measured filter coefficients we account for non-ideal detection efficiency by subtracting the variance of filter coefficients obtained far off mechanical resonances from the variances appearing in the denominator of equation (21). Correlations introduced due to the application of matched filters are accounted for by subtracting correlations of uncoupled oscillators and shot noise in the numerator. The corrected correlation coefficients can be directly compared to coefficients calculated by integrating equation (16) for independently measured experimental parameters for different coupling times (open symbols in Fig. $4 \mathrm{~b}$ and Supplementary Fig. 2 b). Statistical errors are obtained by performing a Fisher transformation on the statistical variance of the sample. To verify the validity of the statistical analysis, we also extract the coefficients of the uncorrelated quantities $\left\langle\operatorname{Re}\left(\hat{Z}_{i}\right) \operatorname{Im}\left(\hat{Z}_{i}\right)\right\rangle$, which are scattered around zero with a standard deviation given by the shaded region of Fig. $4 \mathrm{~b}$.

The observed correlations, averaged over all coupling times, $\bar{r}_{\exp } \approx 0.152$ are slightly reduced from zero-free-parameter theory expectations $\bar{r}_{\text {theo }} \approx 0.176$, probably owing to systematic uncertainties or shot-to-shot fluctuations leading to inhomogeneous averaging. 


\section{References}

35. Purdy, T. P. et al. Tunable cavity optomechanics with ultracold atoms. Phys. Rev. Lett. 105, 133602 (2010).

36. Brahms, N., Botter, T., Schreppler, S., Brooks, D. W. C. \& Stamper-Kurn, D. M. Optical detection of the quantization of collective atomic motion. Phys. Rev. Lett. 108, 133601 (2012).

37. Botter, T., Brooks, D. W. C., Brahms, N., Schreppler, S. \& Stamper-Kurn, D. M. Linear amplifier model for optomechanical systems. Phys. Rev. A 85, 013812 (2012). 38. Kasevich, M. \& Chu, S. Laser cooling below a photon recoil with three-level atoms. Phys. Rev. Lett. 69, 1741-1744 (1992).

39. Buchmann, L. F. \& Stamper-Kurn, D. M. Nondegenerate multimode optomechanics. Phys. Rev. A 92, 013851 (2015). 